\title{
EVALUATION OF VITEK 2 SYSTEM FOR CLINICAL IDENTIFICATION OF CANDIDA SPECIES AND THEIR ANTIFUNGAL SUSCEPTIBILITY TEST
}

\author{
Mohan Sundaram¹, Ram Murugan Navaneethakrishnan² \\ ${ }^{1}$ Assistant Professor, Department of Microbiology, Vinayaka Mission's Medical College, Karaikal. \\ ${ }^{2}$ Senior Assistant Professor, Department of Microbiology, Institute of Microbiology, Madurai Medical College, Madurai.
}

\section{ABSTRACT}

\section{OBJECTIVES}

1. To evaluate the Vitek 2 system for clinical identification of Candida species and their antifungal susceptibility test; 2 . To study the incidence of various types of Candida species in this part of Tamilnadu.

\section{METHODS}

Samples collected from different wards were subjected for culture, isolation and identification of Candida Species and Antifungal Susceptibility testing by Vitek System. Vitek 2 test was carried out in Apollo Specialty Hospital Lab Services, Madurai. The cost per test is Rs. 200 (Subsidized rate). The expenses for the lab tests (Vitek) were borne by the author himself.

\section{RESULTS}

124 samples were collected from urine, sputum, blood, pus and wounds. Candida albicans formed $43 \%$ of the samples. Among the $57 \%$ of Non-Candida albicans, Candida tropicalis formed $42 \%$, Candida krusei formed $6 \%$, Candida guilliermondii formed $4 \%$, Candida inconspicua, Candida parapsilosis, Candida glabrata, Candida rugosa and Candida lusitaniae formed $1 \%$ each. Candida albicans and C. tropicalis showed high sensitivity to Voriconazole, Flucytosine, Amphotericin B and Fluconazole.

\section{CONCLUSION}

Candida tropicalis was identified as the most common Candida non-albicans species. Candida albicans and C. tropicalis showed high sensitivity to Voriconazole, Flucytosine, Amphotericin B and Fluconazole. This study was helpful to treat Candida albicans and Non-Candida albicans species patients accurately and earlier by Vitek method.

\section{KEYWORDS}

Candida Albicans, Non-Candida Albicans, Vitek 2, Chrome Agar.

HOW TO CITE THIS ARTICLE: Sundaram M, Navaneethakrishnan RM. Evaluation of Vitek 2 system for clinical identification of candida species and their antifungal susceptibility test. J. Evolution Med. Dent. Sci. 2016;5(47):2948-2951,

DOI: $10.14260 /$ jemds/2016/687

\section{INTRODUCTION \\ Candida is a genus of yeasts and is the most common cause of fungal infections worldwide. \\ The Candida species are the 4th most common organisms causing blood stream infection and constitute $8 \%$ of all nosocomial infections. ${ }^{1}$ Candidiasis is mainly caused by $\mathrm{C}$. albicans, while there has been striking increase in the frequency with Non C. albicans. The most important species which are considered pathogenic to humans are C. albicans, C. tropicalis, C. kruseii, C. glabrata, C. lusitaniae, Candida dubliniensis, Candida guilliermondii, Candida parapsilosis, Candida kefyr. ${ }^{2}$ HiCrome Candida Differential Agar is a selective and a differential medium, which allows differentiation of Candida species namely C. albicans, C. krusei, C. tropicalis and C. glabrata on the basis of colouration and colony morphology. ${ }^{3}$ The Vitek 2 system accommodates the reagent cards that are incubated and interpreted automatically. 4 The Candida Species sensitivity was observed with Voriconazole, Flucytosine Amphotericin B, Fluconazole, Micafungin and Caspofungin.}

Financial or Other, Competing Interest: None.

Submission 18-04-2016, Peer Review 21-05-2016,

Acceptance 27-05-2016, Published 10-06-2016.

Corresponding Author:

Dr. Ram Murugan Navaneethakrishnan,

\#14/2 Siva Sakthi Nagar,

K. Pudur, Madurai-625007.

E-mail: drsmohan1971@gmail.com,rammddo@yahoo.co.in

DOI: $10.14260 /$ jemds/2016/687

\author{
MATERIALS AND METHODS \\ Type of Study \\ Cross sectional, observational, prospective study.

\section{Settings} \\ The study was conducted in a tertiary care teaching hospital, \\ Vinayaka Mission's Medical College Hospital, Karaikal. Vitek 2 \\ test was carried out in Apollo Specialty Hospital Lab Services, \\ Madurai. The cost per test is Rs. 200 (Subsidized rate). The \\ expenses for the lab tests (Vitek) were borne by the author \\ himself.
}

\section{Sample Size}

124 samples were collected from urine, sputum, blood, pus and wounds.

\section{Study Period}

From March 2015 to March 2016.

\section{Inclusion Criteria}

Patients not treated with any antifungal treatment.

\section{Exclusion Criteria}

Patients already treated with any antifungal treatment.

\section{METHODOLOGY}

Present study was carried out in a tertiary care hospital at Vinayaka Mission's Medical College Hospital, Karaikal, for a period of one year from March 2015 to March 2016. The 
clinical samples isolated from blood, urine, pus, endotracheal secretions and wounds were submitted to the laboratory. Those specimens suspected of fungal infection during the study period were processed. The samples were transported to the laboratory and streaked on Blood Agar medium and plate of Sabouraud Dextrose Agar (SDA) with chloramphenicol and gentamicin.

Blood agar and SDA agar were incubated at $37^{\circ} \mathrm{C}$. After incubating for 48 hours, typical creamy pasty colonies from which "feet" extending out from the margins into the surrounding agar appeared on the blood agar and SDA.

On observing Gram staining from SDA and Blood Agar plates, Candida species appeared as gram positive budding yeast cells with Pseudohyphae, Chlamydospore and blastoconidia.

The germ tube test was done for identifying C. albicans; $0.5 \mathrm{~mL}$ of human serum was taken into a small tube. The colony of yeast was touched with a Pasteur pipette. It was emulsified in the serum and incubated at $37^{\circ} \mathrm{C}$ for 2 hours. A drop of this serum was transferred to a slide for examination. With the coverslip on, slide was examined under a microscope using low and high power objectives.

Candida isolates isolated from blood agar, SDA plates were streaked in CHROMagarTM Candida agar and incubated at $37^{\circ} \mathrm{C}$ for $48 \mathrm{hrs}$. The CHROMagar ${ }^{\mathrm{TM}}$ allows identifying colonies of C. albicans, C. dubliniensis, C. tropicalis and C. krusei by morphology and colour reaction.

C. albicans or C. dubliniensis appeared as green colonies and C. tropicalis as steel blue colonies. C. krusei colonies showed rose colour. The other species formed white to rose colonies.

The speciation of Candida species was done with automated methods. The Vitek 2 was used to identify the Candida Species with Vitek 2 YST Card and anti-fungal susceptibility Card with Vitek 2 AST-YSO7 Card. The cost per test is Rs. 200 (Subsidized rate). The expenses for the lab tests (Vitek) were borne by the author himself.

\section{For Sample Preparation}

$24 \mathrm{hr}$. old culture was tested on the instrument. Vitek 2 compact cassette with labelled barcode was selected. Cassette number was defined. The polystyrene tubes were placed in the cassette; $3 \mathrm{~mL}$ of sterile Vitek saline was taken in the polystyrene tubes. The colonies were isolated from the plate and suspended in Vitek saline ( $3 \mathrm{~mL}$ ) with help of sterile loop. The suspension was made uniform thoroughly. The density of the inoculums was checked by the Densichek plus. McFarland standard (Vitek 2.20 YST and Vitek 2 YST Card) was checked by the Densichek plus provided by the BioMerieux. The cards were then ready for inoculation. Vitek 2.20 YST and Vitek 2 YST Cards pre-inserted were transferred from the respective suspensions into the selected polystyrene tubes. All the inoculums in cassette were ready to be loaded into the instrument in filler section.

\section{Sample Loading}

The cassette was loaded into the Filler section. The door was closed. The Fill on the User Interface Screen was pressed. It was (Filling) completed in 70 seconds cycle. When the filling cycle was finished, the blue indicator light on the instrument flashed. The cassette was loaded into the Load section. (It was done within 10 minutes of removing from the Filling section). Barcodes were scanned and checked against the Maintain
Virtual Cassette electronic Work list. Straws were cut and sealed. Cards were loaded into the carousel. Cassette waste discarded. It was indicated by flashing blue arrow on the instrument. The loading was thus finished.

\section{Result Reading}

In this case, Vitek 2 YST was evaluated using 150 isolates of both commonly and rarely observed species of Candida. The result generated as genus-level, group-level identification, while the Vitek 2 AST-YSO7 Card evaluated the sensitivity and resistance of Amphotericin B, Fluconazole, Micafungin, Caspofungin, Voriconazole, and Flucytosine.

The final profile results were compared with the database and the identification of the unknown organism was obtained. A final identification of "excellent," "very good," "good," "acceptable" or "low discrimination" was considered to be correct.

\section{RESULTS}

\begin{tabular}{|c|c|c|}
\hline Age Group & Total No.=124 & Percentage \\
\hline $1-10$ yrs. & 6 & 5 \\
\hline $11-20$ yrs. & 1 & 1 \\
\hline $21-30$ yrs. & 12 & 9 \\
\hline $31-40$ yrs. & 22 & 18 \\
\hline $41-50$ yrs. & 24 & 19 \\
\hline $51-60$ yrs. & 22 & 18 \\
\hline $61-70$ yrs. & 20 & 16 \\
\hline 71 and above & 17 & 14 \\
\hline \multicolumn{2}{|c|}{ Table 1: $\boldsymbol{A g e}$ Wise Isolation of Candida Species (n=124) }
\end{tabular}

Table 1 shows out of 124 isolates, $6 \%$ isolates were from patients below 20 yrs., $64 \%$ were from patients between 21 $60 \mathrm{yrs}$. and $30 \%$ were from patients above $60 \mathrm{yrs}$.

\begin{tabular}{|c|c|c|}
\hline Sex & $\begin{array}{c}\text { Total No. of } \\
\text { Isolates } \\
(\mathbf{n = 1 2 4 )}\end{array}$ & $\begin{array}{c}\text { Percentage of } \\
\text { Isolates }\end{array}$ \\
\hline Female & 78 & 63 \\
\hline Male & 41 & 33 \\
\hline Male children & 4 & 3 \\
\hline $\begin{array}{c}\text { Female } \\
\text { Children }\end{array}$ & 1 & 1 \\
\hline Total & $\mathbf{1 2 4}$ & $\mathbf{1 0 0}$ \\
\hline \multicolumn{2}{|c|}{ Table 2: Gender Wise Isolation of Candida Species (n=124) } \\
\hline
\end{tabular}

Table 2 shows out of 124 isolates, $63 \%$ of isolates were from females, $33 \%$ were from males, $3 \%$ were from male children and $1 \%$ was from female children.

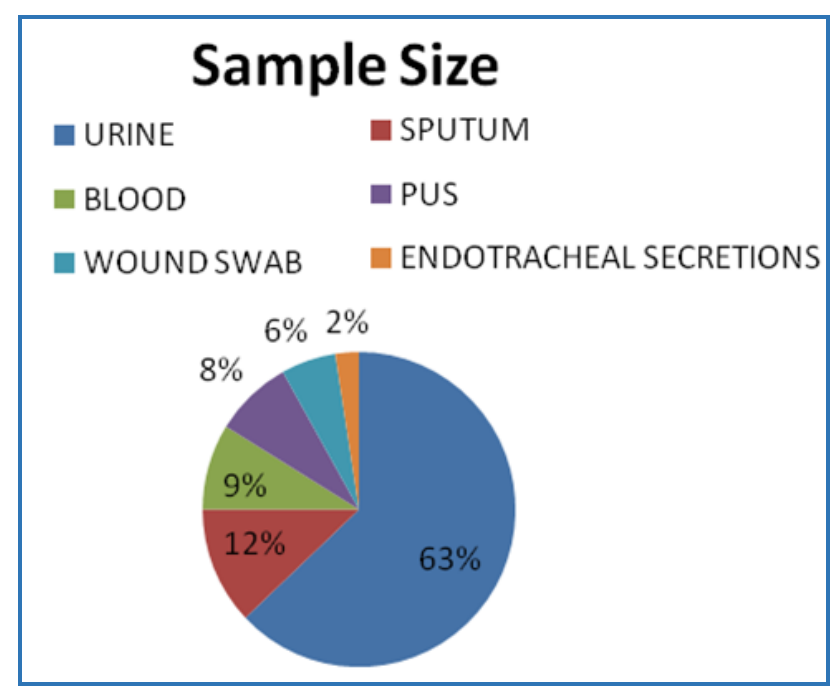

Fig. 1: Sample Wise Isolation $n=124$ 
Fig. 1 shows out of 124 isolates, 63\% were from urine samples, $12 \%$ were from sputum, $9 \%$ were from blood, $8 \%$ were from pus samples, $6 \%$ were from wound swabs and $2 \%$ were from endotracheal secretions.

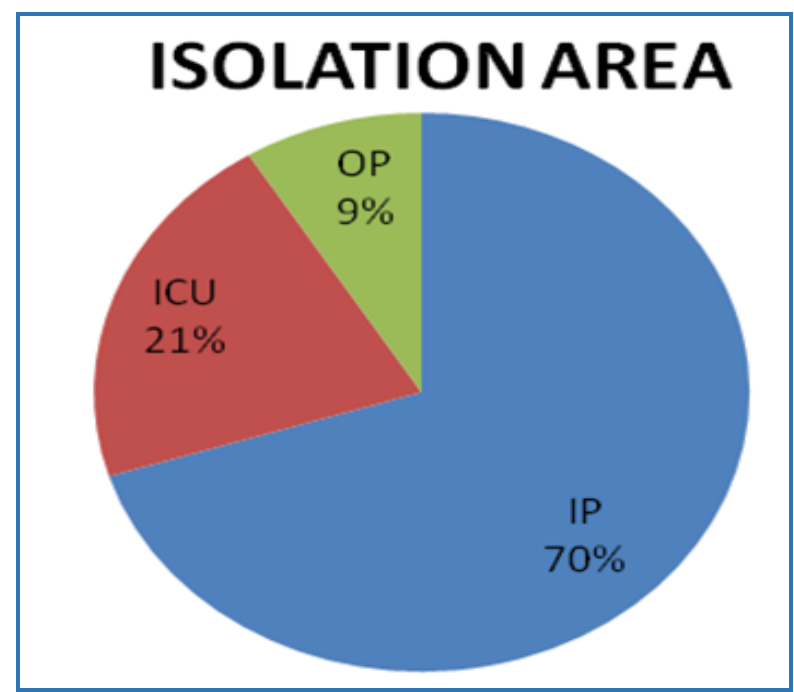

Fig. 2: Area Wise Isolation ( $n=124)$

Fig. 2 shows out of 124 isolates, 70\% were from Inpatients, $21 \%$ were from ICU, $9 \%$ were from Out-patients.

\begin{tabular}{|c|c|c|}
\hline $\begin{array}{l}\text { Candida } \\
\text { Species }\end{array}$ & $\begin{array}{c}\text { Total No. of } \\
\text { Isolates } n=124\end{array}$ & $\begin{array}{l}\text { Percentage of } \\
\text { Isolates }\end{array}$ \\
\hline Candida albicans & 53 & 43 \\
\hline Candida tropicalis & 52 & 42 \\
\hline Candida krusei & 7 & 6 \\
\hline Candida guilliermondii & 5 & 4 \\
\hline Candida inconspicua & 2 & 1 \\
\hline Candida parapsilosis & 1 & 1 \\
\hline Candida glabrata & 1 & 1 \\
\hline Candida rugosa & 1 & 1 \\
\hline Candida lusitaniae & 2 & 1 \\
\hline Total & 124 & 100 \\
\hline
\end{tabular}

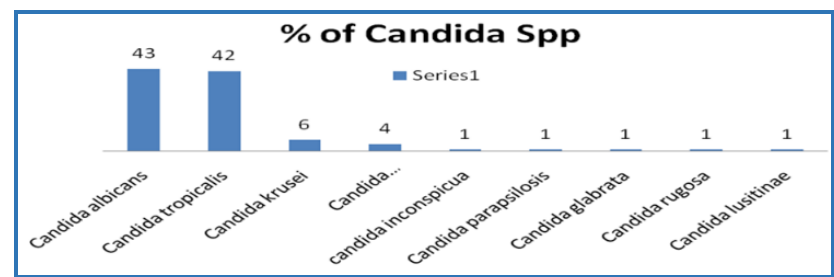

Fig. 3: Isolation of Candida Species ( $n=124)$

Table 3, Fig. 3 shows out of 124 isolates isolated Candida albicans were $43 \%$, Candida tropicalis were $42 \%$, Candida krusei were $6 \%$, Candida guilliermondii were $4 \%$, Candida inconspicua, Candida parapsilosis, Candida glabrata, Candida glabrata, Candida rugosa and Candida lusitaniae were $1 \%$ each.

\begin{tabular}{|c|c|c|c|c|c|c|c|c|c|}
\hline $\begin{array}{l}\text { Candida } \\
\text { Species }\end{array}$ & $\begin{array}{c}\text { C. albicans } \\
(n=53)\end{array}$ & $\begin{array}{l}\text { C. tropi } \\
\text { calis } \\
(n=52)\end{array}$ & $\begin{array}{c}\text { C. guiller } \\
\text { mondi } \\
(n=5)\end{array}$ & $\begin{array}{c}\text { Candida } \\
\text { krusei } \\
(n=7)\end{array}$ & $\begin{array}{c}\text { C. lusitaniae } \\
(n=2)\end{array}$ & $\begin{array}{c}\text { C. parap- } \\
\text { silosis } \\
(n=1)\end{array}$ & $\begin{array}{c}\text { C. rugosa } \\
(n=1)\end{array}$ & $\begin{array}{c}\text { C. } \\
\text { inconspicua } \\
(n=2)\end{array}$ & $\begin{array}{c}\text { C. glabrata } \\
(n=1)\end{array}$ \\
\hline Voriconazole & $49(92 \%)$ & $48(92 \%)$ & $3(60 \%)$ & $6(85 \%)$ & $2(100 \%)$ & $1(100 \%)$ & $1(100 \%)$ & $2(100 \%)$ & $0 \%$ \\
\hline Caspofungin & $40(75 \%)$ & $38(73 \%)$ & $3(60 \%)$ & $6(85 \%)$ & $2(100 \%)$ & $1(100 \%)$ & $1(100 \%)$ & $2(100 \%)$ & $0 \%$ \\
\hline Micafungin & $42(78 \%)$ & $41(79 \%)$ & $3(60 \%)$ & $6(85 \%)$ & $2(100 \%)$ & $1(100 \%)$ & $1(100 \%)$ & $2(100 \%)$ & $0 \%$ \\
\hline Amphotericin B & $47(89 \%)$ & $44(85 \%)$ & $4(80 \%)$ & $6(85 \%)$ & $2(100 \%)$ & $1(100 \%)$ & $1(100 \%)$ & $2(100 \%)$ & $0 \%$ \\
\hline Flucytosine & $49(92 \%)$ & $44(85 \%)$ & $4(80 \%)$ & $1(15 \%)$ & $2(100 \%)$ & $1(100 \%)$ & $1(100 \%)$ & $2(100 \%)$ & $0 \%$ \\
\hline Fluconazole & $44(81 \%)$ & $40(77 \%)$ & $4(80 \%)$ & $1(15 \%)$ & $2(100 \%)$ & $1(100 \%)$ & $1(100 \%)$ & $2(100 \%)$ & $0 \%$ \\
\hline \multicolumn{10}{|c|}{ Table 4: Antifungal Sensitivity Pattern of Candida Isolates ( $n=124)$} \\
\hline
\end{tabular}

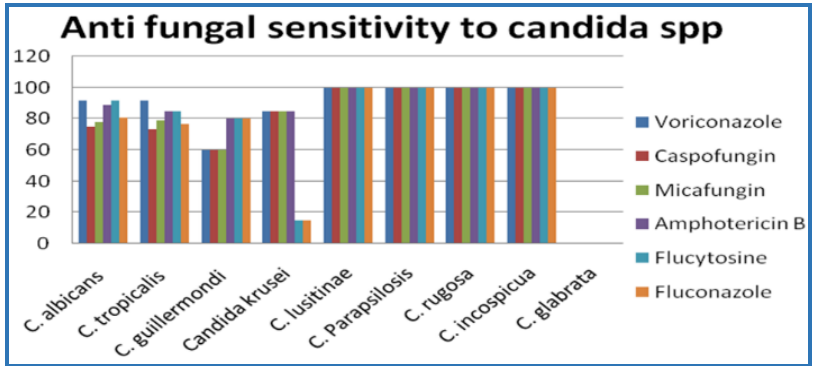

Fig. 4: Antifungal Sensitivity Pattern of Candida Isolates $(n=124)$

Table 4, Fig. 4 shows out of 53 isolates, Candida albicans which showed sensitivity to Voriconazole were 92\%, Flucytosine were 92\%, Amphotericin B were $89 \%$, Fluconazole were $81 \%$, Micafungin were $78 \%$, Caspofungin were $75 \%$.

Out of 52 isolates, Candida tropicalis showed sensitivity to Voriconazole were 92\%, Amphotericin B were 85\%, Flucytosine were $85 \%$, Micafungin were $79 \%$, Fluconazole were $77 \%$, Caspofungin were $73 \%$.
Out of 7 isolates, Candida krusei showed sensitivity to Voriconazole, Caspofungin, Micafungin and Amphotericin B were $85 \%$ each, Flucytosine and Fluconazole were 15\% each.

Out of 5 isolates $\mathrm{C}$. guilliermondii showed sensitivity to Voriconazole, Caspofungin and Micafungin were 60\%; Amphotericin B, Flucytosine and Fluconazole were 80\% each.

Out of 2 isolates C. lusitaniae showed $100 \%$ sensitivity to Caspofungin, Micafungin, Voriconazole, Amphotericin B, Flucytosine and Fluconazole each.

One isolate of C. parapsilosis, C. rugosa, C. inconspicua showed $100 \%$ sensitivity to Caspofungin, Micafungin, Voriconazole, Amphotericin B, Flucytosine and Fluconazole each.

One isolate of Candida glabrata showed $100 \%$ resistance to Caspofungin, Micafungin, Voriconazole, Amphotericin B, Flucytosine and Fluconazole each.

\section{DISCUSSION}

In our study out of 124 isolates isolated, $43 \%$ were Candida albicans and $57 \%$ were Non-Candida albicans. Non-Candida albicans were isolated more in numbers than C. albicans. Rachana Mehta et al, has reported that Candida albicans was 
the commonest species isolated (40.9\%). The isolation of Non-Candida albicans (59.1\%) predominated over Candida albicans (40.9\%). 5 This study correlates with our study.

In our study out of 124 isolates, isolated Candida albicans were $43 \%$. "Among $57 \%$ of Non-Candida albicans, the Candida tropicalis were $42 \%$, Candida krusei were $6 \%$, Candida guilliermondii were 4\%, Candida inconspicua, Candida parapsilosis, Candida glabrata, Candida rugosa and Candida lusitaniae were $1 \%$ each." Dr. Jayalakshmi L et al, 6 has reported that the commonest Candida isolate was $\mathrm{C}$. albicans (31.42\%) followed by C. tropicalis (26.66\%). Other species isolated were C. glabrata $(19.04 \%)$, C. parapsilosis $(10.47 \%)$, C. krusei (5.71\%), C. kefyr (4.76\%) and C. guilliermondii (1.9\%). The most common Non-Candida albicans isolates were C. tropicalis, C. Krusei and C. guilliermondii. This study correlates with our findings.

Candida tropicalis species has emerged as the major Candida non-albicans species (43\%) in our study. Our study correlates with the study by Patel et al (40.9\%), and Kashid et al $(46.25 \%)$ who have also reported Candida tropicalis as the most common Candida non-albicans species. 7,8

In our study, out of 53 isolates Candida albicans which showed sensitivity to Voriconazole were 92\%, Flucytosine were 92\%, Amphotericin B were 89\%, Fluconazole were $81 \%$, Micafungin were $78 \%$, Caspofungin were $75 \%$. Gulgun Yenisehirli et al has reported that all the tested C. albicans isolates were found to be susceptible to amphotericin B; $85 \%$ of the isolates were classified as showing susceptibility to caspofungin. The sensitivity rates of $\mathrm{C}$. albicans isolates to fluconazole and voriconazole were $74 \%$ and $86 \%$ respectively. The Candida albicans showed sensitivity to Amphotericin B, Fluconazole, Voriconazole. ${ }^{9}$ This study bears a good correlation with our study.

In our study, out of 52 isolates Candida tropicalis showed sensitivity to Voriconazole were $92 \%$, Amphotericin B were $85 \%$, Flucytosine were $85 \%$, Micafungin were $79 \%$, Fluconazole were $77 \%$ and Caspofungin were $73 \%$. Pasquale $\mathrm{T}$ et al, has reported that $\mathrm{C}$. tropicalis is usually susceptible to the Voriconazole, Flucytosine and amphotericin B. ${ }^{10}$ In our study Candida tropicalis showed high sensitivity to Voriconazole, amphotericin B and Flucytosine. Thus, our study has similar findings.

In our study, out of 7 isolates Candida krusei showed sensitivity to Voriconazole, Caspofungin, Micafungin and Amphotericin B were 85\% each, Flucytosine and Fluconazole were $15 \%$ each. Orozco AS et al has reported that C. krusei is intrinsically resistant to fluconazole. ${ }^{11}$ Our study correlates well with the study of Orozco AS et al.

In this study, out of 5 isolates $C$. guilliermondii showed sensitivity to Voriconazole, Caspofungin and Micafungin were $60 \%$, Amphotericin B, Flucytosine and Fluconazole were 80\% each. Girmenia $\mathrm{C}$ et al has reported that $\mathrm{C}$. guilliermondii is an uncommon Candida species. This species has reduced susceptibility to Fluconazole. ${ }^{12}$ However, C. guilliermondii is usually susceptible to Amphotericin B.

\section{CONCLUSION}

The conventional methods of identification of Candida species are time consuming and difficult to perform. Candida tropicalis was identified as the most common Candida nonalbicans species. Candida albicans and C. tropicalis showed high sensitivity to Voriconazole, Flucytosine, Amphotericin B and Fluconazole. This study was helpful to treat Candida albicans and non-Candida albicans species patients accurately and earlier by Vitek method. Rapid, accurate susceptibility test results and resistance detection are clinically relevant. This purpose is well served by YST cards with VITEK® 2 instruments.

\section{LIMITATIONS}

This is a single centered study.

\section{ACKNOWLEDGEMENT}

The authors gratefully acknowledge the Professor and Head of Department of Microbiology, Vinayaka Mission's Medical College and Dean Vinayaka Mission's Medical College, Karaikal, to carry out this study. I acknowledge the Head and Medical Officer in charge of Microbiology, Lab services, Apollo Speciality Hospitals, Madurai, to carry out Vitek 2. The help rendered by all technical staff are whole heartedly acknowledged.

\section{REFERENCES}

1. Manolakaki D, Velmahos G, Kourkoumpetis T, et al. Candida infection and colonization among trauma patients. Virulence 2010;1(5):367-75.

2. Krcmery V, Barnes AJ. Non-albicans candida spp. causing fungaemia: pathogenicity and antifungal resistance. J Hospit infec 2002;50(4):243-60.

3. Perry JL, Miller GR, Carr DL. Rapid, colorimetric identification of candida albicans. J Clin Microbiol 1990;28(3):614-5.

4. Giuseppe Valenza, Jörn Strasen, Frauke Schäfer, et al. Evaluation of new colorimetric vitek 2 yeast identification card by use of different source media. Journal Of Clinical Microbiology 2008;46(11):3784-7.

5. Rachana Mehta, Anupama S Wyawahare. Evaluation of hicrome candida differential agar for species identification of candida isolates from various clinical samples. International Journal of Contemporary Medical Research 2016;3(4):1219-22.

6. Jayalakshmi L, Ratna Kumari G, Samson SH. Isolation, speciation and antifungal susceptibility testing of candida from clinical specimens at a tertiary care hospital. Scholars Journal of Applied Medical Sciences (SJAMS) Sch J App Med Sci 2014;2(6E):3193-8.

7. Patel LR, Pethani JD, Bhatia P, et al. Prevalence of candida infection and its antifungal susceptibility pattern in tertiary care hospital, Ahmedabad. Natl J Med Res 2012;2(4):439-41.

8. Kashid RA, Belawadi S, Devi G, et al. Characterization and antifungal susceptibility testing for candida species in a tertiary care hospital. Journal of Health Sciences \& Research 2011;2(2):1-7.

9. Gulgun Yenisehirli, Nermin Bulut, Aydan Yenisehirli, et al. In vitro susceptibilities of candida albicans isolates to antifungal agents in Tokat, Turkey. Jundishapur J Microbiol 2015;8(9):e28057.

10. Pasquale T, Tomada JR, Ghannoun M, et al. Emergence of candida tropicalis resistant to caspofungin. J Antimicrob Chemother 2008;61(1):219.

11. Orozco AS, Higginbotham LM, Hitchcock CA, et al. Mechanism of fluconazole resistance in candida krusei. Antimicrob Agents Chemother 1998;42(10):2645-9.

12. Girmenia C, Pizzarelli G, Cristini F, et al. Candida guilliermondiifungemia in patients with haematologic malignancies. J Clin Microbiol 2006;44(7):2458-64. 